\title{
Gerbil Melanoma
}

National Cancer Institute

\section{Source}

National Cancer Institute. Gerbil Melanoma. NCI Thesaurus. Code C134773.

Melanoma that occurs in a gerbil. 